\title{
Vertical discretizations giving optimal representation of normal modes: General equations of state
}

\author{
J. Thuburn* \\ University of Exeter, Exeter, $U K$ \\ ${ }^{*}$ Correspondence to: Department of Mathematics, College of Engineering, Mathematics and Physical Sciences, University of Exeter, \\ Exeter, EX4 4QF, UK.
}

\begin{abstract}
Previous work has identified a number of vertical discretizations of the nonhydrostatic compressible Euler equations that optimally capture the propagation of acoustic, inertio-gravity, and Rossby waves. Here, that previous work is extend to apply to a general equation of state, making it applicable to a wider range of geophysical fluid systems. It is also shown that several choices of prognostic thermodynamic variables and vertical staggering that were previously thought to be suboptimal can, in fact, give optimal wave propagation when discretized in an appropriate way. The key idea behind constructing these new optimal discretizations is to ensure that their corresponding linear system is equivalent to that of a certain, most fundamental, optimal configuration.
\end{abstract}

Key Words: $\quad$ Wave dispersion; Staggered grid; Numerical model

Received...

\section{Introduction}

The developer of a numerical method for the simulation of geophysical flows is faced with various decisions, including the choice of which thermodynamic variables to predict, and the relative placement of the prognostic variables on the vertical grid, i.e. the vertical staggering. Previous work has identified a number of choices that permit an optimal representation of the propagation of different types of waves: acoustic, inertio-gravity and Rossby waves. Here 'optimal' means that the errors due to vertical averaging in the discrete equations are, in a certain sense, minimized, resulting in a numerical dispersion relation that is as close as possible, for a second-order centred difference scheme on a uniform grid, to the exact dispersion relation. By considering the discrete equations for the vertical momentum and thermodynamic variables, the present work generalizes previous results in two ways: first, to apply to an arbitrary equation of state, and second, to identify a broader class of configurations* for which optimal discretizations may be found; this class allows any two thermodynamic variables from density $\rho$, entropy $\eta$, pressure $p$, and temperature $T$ to be predicted.

Different alternatives for vertical grid staggering were introduced in the early days of numerical modelling of the atmosphere (Charney and Phillips 1953; Lorenz 1960). Grids with potential temperature $\theta$ and vertical velocity staggered relative to horizontal velocity are natural for the representation of quasigeostrophic dynamics, and have become known as CharneyPhillips grids. Grids with vertical velocity staggered relative to potential temperature and horizontal velocity facilitate the design

\footnotetext{
*Here the term 'configuration' will be used to refer to the choice of prognostic variables and their vertical staggering. Any given configuration may be discretized in more than one way, as discussed in this note.
}

of schemes that conserve energy and other properties, and have become known as Lorenz grids.

Systematic analysis of the ability of different vertical grid staggerings to capture wave propagation began with Tokioka (1978), (see also Lesley and Purser 1992; Fox-Rabinovitz 1994, 1996; Liu 2008; Girard et al. 2014). These authors observed that the Charney-Phillips grid gives relatively accurate wave propagation. Tokioka (1978), Fox-Rabinovitz (1994) and Girard et al. (2014) also found some schemes involving prediction of a vertically averaged variable that gave good wave propagation. We do not consider such schemes here because the need to undo the averaging on the predicted variable creates difficulties, and such schemes appear unpopular in nonhydrostatic models. These studies also noted the computational mode of the Lorenz grid (and some other grids): a vertical pattern in the thermodynamic variables that, because of averaging in the discrete equations, spuriously satisfies hydrostatic balance and so is invisible to the dynamics. Some modest improvements in the accuracy of wave propagation can be obtained by increasing the order of accuracy of the discretization beyond two, but this cannot eliminate the Lorenz grid computational mode (Lesley and Purser 1992; Liu 2008). In these studies, however, the analysis is simplified by making the hydrostatic assumption or an anelastic or Boussinesq assumption (or both). Thus, only gravity waves or inertio-gravity waves are considered; acoustic waves are eliminated from consideration.

Thuburn and Woollings (2005) extended this type of analysis to the fully compressible Euler equations, and included a representation of the $\beta$-effect; thus they were able to examine acoustic waves, inertio-gravity waves, and Rossby waves. For height-based, mass-based, and isentropic vertical coordinates, they examined the dispersion relations of a large number of configurations with different choices of thermodynamic variables 
and vertical staggerings. For each vertical coordinate they identified one or two 'optimal' configurations giving the best achievable numerical dispersion relations. They introduced a convenient notation summarizing the choice of predicted variables and their staggering for any configuration. For example $(w \theta, u v p)$ indicates that $w$ and $\theta$ are staggered relative to $u, v$ and $p$, where $w$ is the vertical velocity and $u$ and $v$ are the horizontal velocity components. This particular configuration, a type of CharneyPhillips grid, happens to be optimal in a height-based vertical coordinate.

None of the optimal configurations identified by Thuburn and Woollings (2005) involve prediction of the relevant mass variable (e.g. $\rho$ in a height-based coordinate), suggesting that it would be difficult to achieve mass conservation and optimal wave dispersion at the same time. However, Thuburn and Woollings (2005) only considered the most obvious way of writing various terms in the governing equations. For example, in height coordinates the vertical pressure gradient was written as $\rho^{-1} p_{z}$, where subscript $z$ indicates a partial derivative. Almost simultaneously, Thuburn (2006) and Toy and Randall (2007) noticed that some of the near-optimal configurations identified by Thuburn and Woollings (2005) that do predict the relevant mass variable could be made optimal by writing the pressure gradient in a different way, for example as $C_{p} \theta \Pi_{z}$ for the $(w \theta, u v \rho)$ configuration in a height-based coordinate and the $(w \theta z, u v)$ configuration in a mass-based coordinate. Here, $C_{p}$ is the specific heat capacity at constant pressure and $\Pi=\left(p / p_{0}\right)^{\kappa}$ is the Exner pressure, where $p_{0}$ is a constant reference pressure and $\kappa=R / C_{p}$ with $R$ the gas constant. The $(w \theta, u v \rho)$ configuration using the $C_{p} \theta \nabla \Pi$ form of the pressure gradient is used, for example, in the ENDGame dynamical core (Wood et al. 2014) operational at the Met Office.

These latest results raise two further questions. (i) What other configurations might be modified to have optimal wave dispersion properties by suitably re-expressing the pressure gradient term or other terms in the equations? (ii) While the $\rho^{-1} \nabla p$ form of the pressure gradient is universal, the form $C_{p} \theta \nabla \Pi$ is only applicable for a perfect gas; therefore, are the additional optimal configurations identified by Thuburn (2006) and Toy and Randall (2007) only optimal for a perfect gas, or can they be generalized to other equations of state? This last question is pertinent for using numerical models to simulate atmospheres where there are significant variations in composition, for example from the ground to the thermosphere in Earth's atmosphere (e.g. Akmaev 2011), or for fluids where the equation of state is not well approximated by a perfect gas, such as the ocean, the deep interior of gas giant planets (e.g. Militzer and Hubbard 2013), or laboratory flows (e.g. Read et al. 2000). The present paper addresses these two questions. It is shown that the new optimal $(w \theta, u v \rho)$ height-coordinate configuration found by Thuburn (2006) and Toy and Randall (2007) can remain optimal even with the pressure gradient written as $\rho^{-1} p_{z}$, provided it is evaluated in an appropriate way. This, then, removes the restriction to the perfect gas equation of state. The $(w \eta, u v p)$ configuration, where $\eta$ is the specific entropy, is identified as being the most fundamental optimal configuration. However, for several other configurations, predicting combinations of $\rho, \eta, p$ and $T$, systematic choices can be made in evaluating certain terms so that their corresponding linear systems are equivalent to the optimal linear system; thus these other configurations can also be made optimal.

For brevity we restrict attention to the height-coordinate case; a similar analysis is possible for other vertical coordinates. Section 2 reviews the optimal height-coordinate configurations found by previous authors and shows how they can be modified to express the pressure gradient term as $\rho^{-1} p_{z}$ while remaining optimal. Section 3 notes how these optimal configurations can be generalized for an arbitrary equation of state, and also notes several configurations, previously thought to be suboptimal, that can, in fact, be discretized in such a way as to have optimal wave dispersion.

\section{Analysis for a perfect gas}

We begin with the continuous compressible, nonhydrostatic equations of motion in Cartesian $\beta$-plane geometry for a perfect gas, linearized about a resting, hydrostatic, horizontally uniform basic state (indicated by superscript $(r)$ ). The normal modes of this system are separable with horizontal and temporal dependence proportional to $\exp \{\mathrm{i}(k x+l y-\omega t)\}$, where $k$ and $l$ are horizontal wavenumbers and $\omega$ is the frequency. For such solutions the linearized equations of motion are

$$
\begin{aligned}
-\mathrm{i} \omega u-f v-\frac{i k \beta}{K^{2}} u+\frac{1}{\rho^{(r)}} \mathrm{i} k p & =0, \\
-\mathrm{i} \omega v+f u-\frac{i k \beta}{K^{2}} v+\frac{1}{\rho^{(r)}} \mathrm{i} l p & =0, \\
-\mathrm{i} \omega w+\frac{1}{\rho^{(r)}} p_{z}+g \frac{\rho}{\rho^{(r)}} & =0, \\
-\mathrm{i} \omega \frac{\theta}{\theta^{(r)}}+w A & =0, \\
-\mathrm{i} \omega \frac{p}{\rho^{(r)}}+c^{2}\left(\mathrm{i} k u+\mathrm{i} l v+w_{z}-B w\right) & =0 .
\end{aligned}
$$

Here, $u, v, w, \rho, \theta$ and $p$ are now perturbations to the reference state, $g$ is the gravitational acceleration, $K^{2}=k^{2}+$ $l^{2}$, subscript $z$ indicates a vertical derivative, $A=N^{2} / g$ where $N^{2}=g \theta_{z}^{(r)} / \theta^{(r)}$ is the buoyancy frequency squared, and $B=$ $g / c^{2}$ where $c^{2}=R T^{(r)} /(1-\kappa)$ is the sound speed squared. The property $\rho_{z}^{(r)}=-(A+B) \rho^{(r)}$ will be used to write (7) below. See Thuburn and Woollings (2005) for details of the approximations made in including the $\beta$-effect so as to permit separable solutions.

To close this system we need the linearized equation of state

$$
\frac{1}{c^{2}} \frac{p}{\rho^{(r)}}=\frac{\rho}{\rho^{(r)}}+\frac{\theta}{\theta^{(r)}}
$$

We will also consider the case in which density rather than pressure is predicted; the linearized density evolution equation is

$$
-\mathrm{i} \omega \frac{\rho}{\rho^{(r)}}+\left\{\mathrm{i} k u+\mathrm{i} l v+w_{z}-(A+B) w\right\}=0 .
$$

In the special case of an isothermal reference state $c^{2}$ and $N^{2}$ are constants and it is possible to solve the system (1)-(5), (6) to find the vertical structure of the normal modes and the quintic dispersion relation satisfied by $\omega$ (Thuburn and Woollings 2005).

In the vertically discrete case the vertical derivative terms $p_{z}$ and $w_{z}$ are approximated by finite differences $\delta_{z} p, \delta_{z} w$; we will only look at configurations in which $w$ is staggered relative to $p$, so these derivatives can be approximated by a centred difference over the interval $\Delta z$, which we take to be uniform. Also, some variables may need to be averaged from the levels at which they are defined to the levels at which they are needed. A simple equally-weighted mean will be used, indicated by an overbar.

For an isothermal reference state the discrete linear problem can be solved to find the vertical structure of the normal modes and the corresponding numerical dispersion relation. As expected, the differences between the exact and numerical dispersion relations

${ }^{\ddagger}$ In the rest of this note the term 'equation of state' will be used to refer to any relation that expresses one thermodynamic variable in terms of two others. 
arise from the approximation of the vertical derivatives and the vertical averages. Following Thuburn (2006), the differences can be analysed by noting that the discrete normal modes will have vertical structures such as

$$
p \propto \exp \left(\mathrm{i} m+\frac{1}{H_{p}}\right) z
$$

where $m$ is a vertical wavenumber and $H_{p}$ is a height scale, which might be negative, for the pressure perturbation (other fields will have their own height scale, but all will have the same $m$ ). The finite difference vertical derivative of $p$ is then equal to the exact vertical derivative of $p$ multiplied by a factor

$$
\begin{aligned}
& S_{(p)}=\frac{1}{\left(\mathrm{i} m+1 / H_{p}\right) \Delta z} \\
& \times\left[\exp \left\{\left(\mathrm{i} m+\frac{1}{H_{p}}\right) \frac{\Delta z}{2}\right\}-\exp \left\{-\left(\mathrm{i} m+\frac{1}{H_{p}}\right) \frac{\Delta z}{2}\right\}(9)\right.
\end{aligned}
$$

(this corrects an error in equation (26) of Thuburn 2006), while the vertical average of $p$ is equal to the exact value of $p$ multiplied by a factor

$C_{(p)}=\frac{1}{2}\left[\exp \left\{\left(\mathrm{i} m+\frac{1}{H_{p}}\right) \frac{\Delta z}{2}\right\}+\exp \left\{-\left(\mathrm{i} m+\frac{1}{H_{p}}\right) \frac{\Delta z}{2}\right\}\right]$.

Analogous factors arise for derivatives and averages of other fields.

For realistic reference temperature profiles the normal modes can no longer be found analytically in either the continuous or the discrete case. Nevertheless, useful understanding can be derived by considering the limit of large vertical wavenumber, in which variations in reference state profiles are assumed to be small on the scale of the vertical wavelength. We can then take $c^{2}$ and $N^{2}$ as locally approximately constant, and the dispersion relation holds approximately using the local values of $c^{2}$ and $N^{2}$. (Essentially we are making a WKB approximation, e.g. Lighthill (1978).) Restricting attention to the large vertical wavenumber case is not a serious limitation on the analysis because this is precisely the case for which discretization errors will be greatest. In this case the inverse height scale $1 / H_{p}$ becomes negligible compared with $m$ and the factors $S_{(p)}$ and $C_{(p)}$ are approximated by

$$
S=\frac{2}{m \Delta z} \sin \left(\frac{m \Delta z}{2}\right) ; \quad C=\cos \left(\frac{m \Delta z}{2}\right)
$$

Thus, the numerical dispersion relation acquires some factors of $S$ arising from the approximation of vertical derivatives, along with, possibly, some factors of $C$ arising from vertical averages. For the smallest resolvable vertical wavelength $m \Delta z=\pi$ we have $S=2 / \pi$, giving a moderate distortion of those terms arising from vertical derivatives. On the other hand, $C=0$ in this limit, so any terms in the dispersion relation that have picked up factors of $C$ will be lost and the wave propagation can be significantly distorted.

For present purposes it is not necessary to compute the numerical dispersion relation. It is sufficient to examine the discrete linear system to see which terms are vertically averaged. Any scheme whose linear system is equivalent to that of the optimal $(w \theta, u v p)$ configuration will have the same dispersion relation and hence will itself be optimal. To this end, the optimal height-coordinate configurations identified by Thuburn and Woollings (2005), Thuburn (2006) and Toy and Randall (2007) are first briefly reviewed.

\subsection{The $(w \theta, u v p)$ configuration}

We will only consider configurations in which $w$ is staggered relative to $u$ and $v$, and $p$ is defined primarily at $u-v$ levels. Thus, no vertical averaging is needed in the discrete linearized $u$ and $v$ equations, which therefore look exactly like their continuous counterparts (1) and (2). Henceforth we focus on the $w$ equation, the prognostic equations for the thermodynamic variables, and the equation of state. For the optimal $(w \theta, u v p)$ configuration a vertically averaged $w$ appears in the pressure equation. Also, a value of $\rho$ is needed at $w$ levels (indicated by superscript $(w)$ ) in the $w$ equation; this is obtained using the equation of state evaluated at $w$-levels, which involves a vertically averaged $p$.

$$
\begin{aligned}
-\mathrm{i} \omega w+\frac{1}{\rho^{(r)}} \delta_{z} p+g \frac{\rho^{(w)}}{\rho^{(r)}} & =0, \\
-\mathrm{i} \omega \frac{\theta}{\theta^{(r)}}+w A & =0, \\
-\mathrm{i} \omega \frac{p}{\rho^{(r)}}+c^{2}\left(\mathrm{i} k u+\mathrm{i} l v+\delta_{z} w-B \bar{w}\right) & =0, \\
\frac{1}{c^{2}} \frac{\bar{p}}{\rho^{(r)}}=\frac{\rho^{(w)}}{\rho^{(r)}}+\frac{\theta}{\theta^{(r)}} . &
\end{aligned}
$$

Using (15) to eliminate $\rho^{(w)},(12)$ becomes

$$
-\mathrm{i} \omega w+\frac{1}{\rho^{(r)}}\left(\delta_{z} p+B \bar{p}\right)-g \frac{\theta}{\theta^{(r)}}=0 .
$$

Thus, the discrete linear system contains two vertically averaged terms: $\bar{w}$ in (14) and $\bar{p}$ in (16). However, in both cases the vertically averaged term appears alongside a vertical derivative of the same quantity, and in the limit of large vertical wavenumber, which is when the errors due to averaging will be most serious, the averaged term is dominated by the vertical derivative term, so the averaging errors remain negligible after all.

\subsection{The $(w \theta, u v \rho)$ configuration}

Next we look at three versions of the $(w \theta, u v \rho)$ configuration, beginning with the near optimal version examined by Thuburn and Woollings (2005):

$$
\begin{aligned}
-\mathrm{i} \omega w+\frac{1}{\rho^{(r)}} \delta_{z} p+g \frac{\rho^{(w)}}{\rho^{(r)}} & =0, \\
-\mathrm{i} \omega \frac{\theta}{\theta^{(r)}}+w A & =0, \\
-\mathrm{i} \omega \frac{\rho}{\rho^{(r)}}+\left\{\mathrm{i} k u+\mathrm{i} l v+\delta_{z} w-(A+B) \bar{w}\right\} & =0, \\
\frac{1}{c^{2}} \frac{p}{\rho^{(r)}}=\frac{\rho}{\rho^{(r)}}+\frac{\bar{\theta}}{\theta^{(r)}} . &
\end{aligned}
$$

In this version the equation of state is evaluated at $u-v$ levels to provide a value of $p$, and $\rho^{(w)}$ is evaluated as a simple average of the predicted value $\rho^{(w)}=\bar{\rho}$. Using (20), (19) and (18) may be combined to obtain the implied evolution equation for $p$; it is identical to (14). However, using (20) to eliminate $\bar{\rho}$ in (17) leaves

$$
-\mathrm{i} \omega w+\frac{1}{\rho^{(r)}}\left(\delta_{z} p+B \bar{p}\right)-g \frac{\overline{\bar{\theta}}}{\theta^{(r)}}=0 .
$$

Thus, the linear system for this version is almost the same as the optimal $(w \theta, u v p)$ configuration except for the double averaging of $\theta$ in (21). This double averaging of the buoyancy term effectively reduces the static stability seen by the scheme and leads to a retardation of higher internal Rossby modes for long horizontal wavelengths and of higher internal gravity modes for short horizontal wavelengths (Thuburn 2006). 
As an alternative, Thuburn (2006) and Toy and Randall (2007) proposed to evaluate the pressure gradient term not as $\rho^{-1} \nabla p$ but as $C_{p} \theta \nabla \Pi$. Using the linearized definition of the Exner pressure $\Pi / \Pi^{(r)}=\kappa p / p^{(r)}$, the linearized vertical momentum equation becomes

$$
-\mathrm{i} \omega w+\frac{1}{\rho^{(r)}}\left(\delta_{z} p+B \bar{p}\right)-g \frac{\theta}{\theta^{(r)}}=0 .
$$

The linear system is now identical to that for the optimal $(w \theta, u v p)$ configuration, and thus this system, too, has optimal dispersion relation. The pressure gradient term has been constructed in such a way that the buoyancy contribution from $\theta$ remains unaveraged.

We can exploit this insight to obtain optimal wave dispersion while retaining the $\rho^{-1} \nabla p$ form of the pressure gradient term. The key idea is to apply the equation of state twice, first at $u-v$ levels (20) to obtain $p$ from $\rho$ and $\bar{\theta}$, and again at $w$ levels (15) to obtain $\rho^{(w)}$ from $\bar{p}$ and $\theta$. At first sight this appears to introduce a double averaging of $\theta$ in the $w$ equation. However, if we derive the evolution equation for $w$ and the implied evolution equation for $p$, regarding $u, v, w, \theta$ and $p$ as the unknowns, then the linear system is again identical to that for the optimal $(w \theta, u v p)$ configuration, so optimal wave dispersion is obtained. To apply this idea in the full nonlinear equations it is simply necessary to apply the full equation of state at $u-v$ levels to obtain $p=p(\rho, \bar{\theta})$ and again at $w$ levels to obtain $\rho^{(w)}=\rho(\bar{p}, \theta)$.

\section{Analysis for a general equation of state, and some new optimal discretizations}

For a general equation of state the perfect gas definition of potential temperature $\theta=T\left(p_{0} / p\right)^{\kappa}$ no longer applies, and the Exner pressure $\Pi=\left(p / p_{0}\right)^{\kappa}$ loses its significance. (In fact a materially conserved potential temperature may be defined for a general equation of state-see e.g. Feistel et al. (2010). It is a function of entropy and could be used in place of entropy below.) However, we have seen already that the pressure gradient term may be written as $\rho^{-1} p_{z}$ without sacrificing optimal wave dispersion; it is not necessary to use the $C_{p} \theta \nabla \Pi$ form. It is then convenient to use entropy $\eta$ in place of $\theta$ as one of the thermodynamic variables. The continuous linearized equations remain (1)-(5) except that (4) is replaced by

$$
-\mathrm{i} \omega Q \eta+w A=0
$$

where

$$
Q=-\left.\frac{\partial \ln \rho}{\partial \eta}\right|_{p} ^{(r)}
$$

(the subscript $p$ indicates that the partial derivative is taken at constant pressure). A linearized equation of state can be obtained by differentiating the nonlinear expression $\rho=\rho(\eta, p)$, giving

$$
\rho=\left.\frac{\partial \rho}{\partial \eta}\right|_{p} \eta+\left.\frac{\partial \rho}{\partial p}\right|_{\eta} p
$$

or, dividing by $\rho^{(r)}$,

$$
\frac{1}{c^{2}} \frac{p}{\rho^{(r)}}=\frac{\rho}{\rho^{(r)}}+Q \eta
$$

where

$$
c^{2}=\left.\frac{\partial p}{\partial \rho}\right|_{\eta} ^{(r)}
$$

is the sound speed squared for a general compressible fluid. The buoyancy frequency squared is given by the general expression

$$
\frac{N^{2}}{g}=-\frac{\rho_{z}^{(r)}}{\rho^{(r)}}-\frac{g}{c^{2}}
$$

(e.g. IOC et al. 2010), or, writing

$$
\frac{\rho_{z}^{(r)}}{\rho^{(r)}}=\left.\frac{\partial \ln \rho}{\partial \eta}\right|_{p} ^{(r)} \eta_{z}^{(r)}+\left.\frac{\partial \ln \rho}{\partial p}\right|_{\eta} ^{(r)} p_{z}^{(r)}
$$

and using hydrostatic balance,

$$
N^{2}=g Q \eta_{z}^{(r)}
$$

We will also consider using temperature $T$ as one of the predicted variables; its linearized tendency equation is

$$
-\mathrm{i} \omega T+w T_{z}^{(r)}+\left.\rho^{(r)} c^{2} \frac{\partial T}{\partial p}\right|_{\eta} ^{(r)}\left(\mathrm{i} k u+\mathrm{i} l v+w_{z}\right)=0 .
$$

For a general equation of state there is no longer any guarantee that a hydrostatic reference state with constant $c^{2}$ and $N^{2}$ exists and therefore that exact analytical normal modes can be found. Nevertheless, we can proceed as in section 2 by restricting attention to large vertical wavenumber $m$ so that $c^{2}$ and $N^{2}$ are approximately constant on the scale of the waves. The continuous dispersion relation is then identical to that in the perfect gas case, using the local values of $c^{2}$ and $N^{2}$. In the discrete case, consistent with this approximation, we will assume that reference state profiles, including $c^{2}$ and $N^{2}$, are smooth on the grid scale, so that multiplicative factors of reference state quantites can be moved inside or outside averaging operators with negligible error; e.g. $\overline{B \bar{w}} \approx B \overline{\bar{w}}$. The definitions $A=N^{2} / g$ and $B=g / c^{2}$ are retained, and it remains true that $\rho_{z}^{(r)}=-(A+B) \rho^{(r)}$, even for a general equation of state.

In the remainder of this section we look at a number of alternative configurations. In several cases the most obvious discretization is not optimal; however, in each case we can identify why it fails to be optimal and, using the ideas introduced above, modify the discretization to make it optimal.

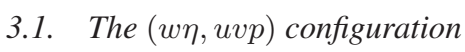

This configuration is the straightforward generalization to an arbitrary equation of state of the configuration discussed in section 2.1. Using (26) evaluated at $w$ levels to eliminate $\rho^{(w)}$ from the $w$ equation, the discrete linear system reduces to

$$
\begin{aligned}
-\mathrm{i} \omega w+\frac{1}{\rho^{(r)}}\left(\delta_{z} p+B \bar{p}\right)-g Q \eta & =0, \\
-\mathrm{i} \omega Q \eta+w A & =0, \\
-\mathrm{i} \omega \frac{p}{\rho^{(r)}}+c^{2}\left(\mathrm{i} k u+\mathrm{i} l v+\delta_{z} w-B \bar{w}\right) & =0 .
\end{aligned}
$$

This scheme is arguably the most fundamental of the optimal schemes, since it emerges naturally from an obvious discretization once the $(w \eta, u v p)$ configuration is specified. As for the configuration of section 2.1, the only averaged terms that appear do so in combination with vertical derivatives of the same quantity, so the errors due to averaging never dominate the dispersion relation. It is also consistent with the heuristic arguments of Thuburn et al. (2002) in terms of the vertical structures of normal modes. The strategy for finding optimal versions of other configurations will be to manipulate them so that their discrete linear system is equivalent to this one.

\subsection{The $(w \eta, u v \rho)$ configuration}

This configuration is the generalization to an arbitrary equation of state of the configuration discussed in section 2.2. Paralleling the discussion there, the equation of state must be applied at $u-v$ 
levels to find $p$ and again at $w$ levels to find $\rho^{(w)}$ :

$$
\begin{aligned}
-\mathrm{i} \omega w+\frac{1}{\rho^{(r)}} \delta_{z} p+g \frac{\rho^{(w)}}{\rho^{(r)}} & =0,-i \omega Q \eta+w A=0, \\
-\mathrm{i} \omega \frac{\rho}{\rho^{(r)}}+\left\{\mathrm{i} k u+\mathrm{i} l v+\delta_{z} w-(A+B) \bar{w}\right\} & =0 \\
\frac{1}{c^{2}} \frac{p}{\rho^{(r)}}=\frac{\rho}{\rho^{(r)}}+Q \bar{\eta}, & \\
\frac{1}{c^{2}} \frac{\bar{p}}{\rho^{(r)}}=\frac{\rho^{(w)}}{\rho^{(r)}}+Q \eta &
\end{aligned}
$$

Eliminating $\rho^{(w)}$ from the $w$ equation leaves (32), while combining (36) and (37) to give the implied $p$ equation leaves (34). Thus, this discrete linear system reduces to the optimal one (32)-(34).

\subsection{The $(w \rho, u v p)$ configuration}

In this configuration $\rho$ is predicted at $w$ levels; the notation $\rho^{(w)}$ is used to keep this clear. The linearization of one obvious discretization (in which the density equation is written as $D \rho / D t+\rho \nabla \cdot \mathbf{u}=0)$ is

$$
\begin{aligned}
-\mathrm{i} \omega w+\frac{1}{\rho^{(r)}} \delta_{z} p+g \frac{\rho^{(w)}}{\rho^{(r)}} & =0, \\
-\mathrm{i} \omega \frac{p}{\rho^{(r)}}+c^{2}\left(\mathrm{i} k u+\mathrm{i} l v+\delta_{z} w-B \bar{w}\right) & =0, \\
-\mathrm{i} \omega \frac{\rho^{(w)}}{\rho^{(r)}}+\left\{\mathrm{i} k \bar{u}+\mathrm{i} l \bar{v}+\delta_{z} \bar{w}-(A+B) w\right\} & =0 .
\end{aligned}
$$

Substituting the equation of state

$$
\frac{1}{c^{2}} \frac{\bar{p}}{\rho^{(r)}}=\frac{\rho^{(w)}}{\rho^{(r)}}+Q \eta
$$

in (40) gives (32). Using it again to construct the implied evolution equation for $\eta$ gives

$$
-\mathrm{i} \omega Q \eta+(A+B) w-B \overline{\bar{w}}=0 .
$$

Thus the linear system differs from the optimal one through the appearance of $B(w-\overline{\bar{w}})$ in (44). The effect of this term is to exaggerate the effect of the buoyancy frequency for large vertical wavenumbers and thus to lead to spuriously fast Rossby waves, as found by Thuburn and Woollings (2005) for the perfect gas case.

This configuration could be made optimal if we could replace the discrete linearized density equation by

$$
-\mathrm{i} \omega \frac{\rho^{(w)}}{\rho^{(r)}}+\left\{\mathrm{i} k \bar{u}+\mathrm{i} l \bar{v}+\delta_{z} \bar{w}-A w-B \overline{\bar{w}}\right\}=0 .
$$

Then the $B w$ terms would cancel in the implied evolution equation for $\eta$ and it would agree with the optimal one (33). One way to achieve this would be to write the full nonlinear density equation as

$$
\frac{D \rho}{D t}=-\rho Q \frac{D \eta}{D t}+\frac{1}{c^{2}} \frac{\overline{D p}}{D t}
$$

and substitute the obvious (and optimal) discretizations of the $\eta$ and $p$ equations, with the latter averaged to $w$ levels.

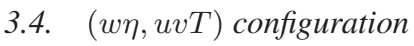

The discrete linearized prognostic equations for this configuration are

$$
\begin{aligned}
-\mathrm{i} \omega w+\frac{1}{\rho^{(r)}} \delta_{z} p+g \frac{\rho^{(w)}}{\rho^{(r)}} & =0, \\
-\mathrm{i} \omega Q \eta+w A & =0, \\
-\mathrm{i} \omega T+\bar{w} T_{z}^{(r)} & \\
+\left.\rho^{(r)} c^{2} \frac{\partial T}{\partial p}\right|_{\eta} ^{(r)}\left(\mathrm{i} k u+\mathrm{i} l v+\delta_{z} w\right) & =0 .
\end{aligned}
$$

If we use

$$
p=\left.\frac{\partial p}{\partial \eta}\right|_{T} ^{(r)} \bar{\eta}+\left.\frac{\partial p}{\partial T}\right|_{\eta} ^{(r)} T
$$

to derive the implied evolution equation for $p$, we find it agrees with the optimal form (34). Next consider the $w$ equation. If we eliminate $\rho^{(w)}$ in the most obvious way using

$$
\rho^{(w)}=\left.\frac{\partial \rho}{\partial \eta}\right|_{T} ^{(r)} \eta+\left.\frac{\partial \rho}{\partial T}\right|_{\eta} ^{(r)} \bar{T}
$$

then the $w$ equation becomes

$$
-\mathrm{i} \omega w+\frac{1}{\rho^{(r)}} \delta_{z} p+\frac{g}{\rho^{(r)}}\left(\left.\frac{\partial \rho}{\partial \eta}\right|_{T} ^{(r)} \eta+\left.\frac{\partial \rho}{\partial T}\right|_{\eta} ^{(r)} \bar{T}\right)=0 .
$$

Rewriting this in terms of $p$ and $\eta$ using (50) gives

$$
-\mathrm{i} \omega w+\frac{1}{\rho^{(r)}}\left(\delta_{z} p+B \bar{p}\right)-g Q \eta+\left.\frac{B}{\rho^{(r)}} \frac{\partial p}{\partial \eta}\right|_{T} ^{(r)}(\eta-\overline{\bar{\eta}})=0 .
$$

This differs from the optimal form through the inclusion of the last term on the left hand side. Once again, the effect of the error is to exaggerate the buoyancy frequency for large vertical wavenumbers leading to spuriously fast Rossby waves, consistent with the $(w \theta, u v T)$ configuration examined by Thuburn and Woollings (2005) and Thuburn (2006).

An optimal version of this configuration can be obtained by not using (51) to eliminate $\rho^{(w)}$ but, rather, using (50) to obtain $p$ and then (39) to obtain $\rho^{(w)}$. The $w$ equation is then of the optimal form (32). For the full nonlinear equations one would use the equation of state first at $u$-v levels to obtain $p=p(T, \bar{\eta})$, then again at $w$ levels to obtain $\rho^{(w)}=\rho(\bar{p}, \eta)$.

\subsection{The (wT, uvp) configuration}

Using an obvious discretization of the $T$ equation, the discrete linearized prognostic equations for this configuration are

$$
\begin{aligned}
-\mathrm{i} \omega w+\frac{1}{\rho^{(r)}} \delta_{z} p+g \frac{\rho^{(w)}}{\rho^{(r)}} & =0, \\
-\mathrm{i} \omega \frac{p}{\rho^{(r)}}+c^{2}\left(\mathrm{i} k u+\mathrm{i} l v+\delta_{z} w-B \bar{w}\right) & =0, \\
-\mathrm{i} \omega T+w T_{z}^{(r)} & \\
+\left.\rho^{(r)} c^{2} \frac{\partial T}{\partial p}\right|_{\eta} ^{(r)}\left(\mathrm{i} k \bar{u}+\mathrm{i} l \bar{v}+\delta_{z} \bar{w}\right) & =0 .
\end{aligned}
$$

Using

$$
\eta=\left.\frac{\partial \eta}{\partial T}\right|_{p} ^{(r)} T+\left.\frac{\partial \eta}{\partial p}\right|_{T} ^{(r)} \bar{p}
$$

to obtain $\eta$ and then (39) to obtain $\rho^{(w)}$ puts (54) in the optimal form (32). However, using (57) again to obtain the implied 
evolution equation for $\eta$ gives

$$
-\mathrm{i} \omega Q \eta+w A-\left.Q \frac{\partial \eta}{\partial p}\right|_{T} ^{(r)} p_{z}^{(r)}(w-\overline{\bar{w}})=0 .
$$

This differs from the optimal form through the inclusion of the last term on the left hand side. For a perfect gas the coefficient $Q \partial \eta /\left.\partial p\right|_{T} ^{(r)} p_{z}^{(r)}$ is positive, so the effect of the error in (58) is to underestimate the buoyancy frequency, leading to spuriously slow Rossby waves, in agreement with Thuburn and Woollings (2005).

An optimal version of this configuration can be obtained by modifying the discretization of the $T$ equation so that its linearized form becomes

$$
\begin{aligned}
-\mathrm{i} \omega T & +\left.w \frac{\partial T}{\partial \eta}\right|_{p} ^{(r)} \eta_{z}^{(r)}+\left.\overline{\bar{w}} \frac{\partial T}{\partial p}\right|_{\eta} ^{(r)} p_{z}^{(r)} \\
& +\left.\rho^{(r)} c^{2} \frac{\partial T}{\partial p}\right|_{\eta} ^{(r)}\left(\mathrm{i} k \bar{u}+\mathrm{i} l \bar{v}+\delta_{z} \bar{w}\right)=0 .
\end{aligned}
$$

Then the implied evolution equation for $\eta$ reduces to the optimal form (33). One way to achieve this would be to write the full nonlinear temperature equation as

$$
\frac{D T}{D t}=\left.\frac{\partial T}{\partial \eta}\right|_{p} ^{(r)} \frac{D \eta}{D t}+\left.\frac{\partial T}{\partial p}\right|_{\eta} ^{(r)} \frac{\overline{D p}}{D t}
$$

and substitute the obvious (and optimal) discretizations of the $\eta$ and $p$ equations, with the latter averaged to $w$ levels.

\subsection{The (wT, uv $\rho)$ configuration}

All of the configurations considered so far in this section predict either $p$ or $\eta$ (or both). Their perfect gas equivalents, using the most obvious discretizations, were classified by Thuburn and Woollings (2005) as either optimal or near optimal; for the near optimal configurations we have seen how an optimal scheme can be obtained by a relatively straightforward modification. In the final two configurations neither $p$ nor $\eta$ is predicted, and Thuburn and Woollings (2005) placed them in a large class of 'problematic' schemes. Nevertheless, optimal discretizations can be constructed based on the lessons learned from sections 3.1-3.5.

For the $(w T, u v \rho)$ configuration the optimal linear discretization is

$$
\begin{aligned}
& -\mathrm{i} \omega w+\frac{1}{\rho^{(r)}} \delta_{z} p+g \frac{\rho^{(w)}}{\rho^{(r)}}=0, \\
& -\mathrm{i} \omega \frac{\rho}{\rho^{(r)}}+\left\{\mathrm{i} k u+\mathrm{i} l v+\delta_{z} w-(A+B) \bar{w}\right\}=0, \\
& -\mathrm{i} \omega T+\left.w \frac{\partial T}{\partial \eta}\right|_{p} ^{(r)} \eta_{z}^{(r)}+\left.\overline{\bar{w}} \frac{\partial T}{\partial p}\right|_{\eta} ^{(r)} p_{z}^{(r)} \\
& +\left.\rho^{(r)} c^{2} \frac{\partial T}{\partial p}\right|_{\eta} ^{(r)}\left(\mathrm{i} k \bar{u}+\mathrm{i} l \bar{v}+\delta_{z} \bar{w}\right)=0, \\
& \eta=\left.\frac{\partial \eta}{\partial T}\right|_{p} ^{(r)} T+\left.\frac{\partial \eta}{\partial p}\right|_{T} ^{(r)} \bar{p} \\
& p=\left.\frac{\partial p}{\partial \eta}\right|_{\rho} ^{(r)} \bar{\eta}+c^{2} \rho
\end{aligned}
$$

along with (39). Note that the equation of state is now used three times: (i) to relate the prognostic thermodynamic variable at $w$ levels (in this case $T$ ) to $\eta$ and $\bar{p}$; (ii) to relate the prognostic thermodynamic variable at $u-v$ levels (in this case $\rho$ ) to $\bar{\eta}$ and $p$; and (iii) to express $\rho^{(w)}$ in terms of $\eta, \bar{p}$. In addition, the prognostic equations for $\rho$ and $T$ take the same form they do in the optimal schemes discussed above.

The two versions of the equation of state (64) and (65) must be solved simultaneously to obtain $p$ and $\eta$ :

$$
\mathcal{L}_{1}(p) \equiv p-\left.\left.\frac{\partial p}{\partial \eta}\right|_{\rho} ^{(r)} \frac{\partial \eta}{\partial p}\right|_{T} ^{(r)} \overline{\bar{p}}=\left.\left.\frac{\partial p}{\partial \eta}\right|_{\rho} ^{(r)} \frac{\partial \eta}{\partial T}\right|_{p} ^{(r)} \bar{T}+c^{2} \rho
$$

and

$$
\mathcal{L}_{1}(\eta)=\left.c^{2} \frac{\partial \eta}{\partial p}\right|_{T} ^{(r)} \bar{\rho}+\left.\frac{\partial \eta}{\partial T}\right|_{p} ^{(r)} T .
$$

Thus, a vertical tridiagonal system must be solved to obtain either $p$ or $\eta$ (the other can then be obtained by back-substitution).

We can confirm that the implied tendency equations for $p$ and $\eta$ are of the optimal form. Substituting (62) and (63) in (66), after some maniputation, leads to

$$
-\mathrm{i} \omega \frac{\mathcal{L}_{1}(p)}{\rho^{(r)}}+c^{2} \mathcal{L}_{1}\left(\mathrm{i} k u+\mathrm{i} l v+\delta_{z} w-B \bar{w}\right)=0 .
$$

Provided $\mathcal{L}_{1}$ is invertible, this is equivalent to (34). The operator $\mathcal{L}_{1}$ is guaranteed be invertible provided its tridiagonal matrix representation is diagonally dominant, which, in turn, will be true provided

$$
\left.\left.\frac{\partial p}{\partial \eta}\right|_{\rho} ^{(r)} \frac{\partial \eta}{\partial p}\right|_{T} ^{(r)}<1
$$

It may be verified that this condition does hold for a perfect gas.

A similar argument can be used to show that the implied $\eta$ tendency equation is equivalent to (33). Alternatively, given that the $p$ and $T$ tendency equations are of the same form as the optimal version of the $(w T, u v p)$ configuration, it immediately follows that the implied $\eta$ tendency equation is of the optimal form.

To apply this scheme to the full nonlinear equations it will be necessary to solve simultaneously the nonlinear versions of (64) and (65):

$$
\begin{aligned}
\eta & =\eta(T, \bar{p}), \\
p & =p(\bar{\eta}, \rho) .
\end{aligned}
$$

This is a nonlinear and nonlocal problem. One obvious approach would be to use a Newton method; at each Newton iteration a linear system like (66) would need to be solved. With a good first guess only a small number of Newton iterations would be required. Nevertheless, this clearly involves more work and greater complexity than a simple local evaluation of the equation of state.

\subsection{The $(w \rho, u v T)$ configuration}

To complete the set, the optimal version of the $(w \rho, u v T)$ configuration is given by

$$
\begin{aligned}
& -\mathrm{i} \omega w+\frac{1}{\rho^{(r)}} \delta_{z} p+g \frac{\rho^{(w)}}{\rho^{(r)}}=0, \\
& -\mathrm{i} \omega \frac{\rho^{(w)}}{\rho^{(r)}}+\left\{\mathrm{i} k \bar{u}+\mathrm{i} l \bar{v}+\delta_{z} \bar{w}-A w-B \overline{\bar{w}}\right\}=0, \\
& -\mathrm{i} \omega T+\bar{w} T_{z}^{(r)} \\
& +\left.\rho^{(r)} c^{2} \frac{\partial T}{\partial p}\right|_{\eta} ^{(r)}\left(\mathrm{i} k u+\mathrm{i} l v+\delta_{z} w\right)=0 \\
& \eta=\left.\frac{\partial \eta}{\partial \rho}\right|_{p} ^{(r)} \rho+\left.\frac{\partial \eta}{\partial p}\right|_{\rho} ^{(r)} \bar{p} \\
& p=\left.\frac{\partial p}{\partial \eta}\right|_{T} ^{(r)} \bar{\eta}+\left.\frac{\partial p}{\partial T}\right|_{\eta} ^{(r)} T .
\end{aligned}
$$


Similarly to the $(w T, u v \rho)$ configuration, the equation of state is used three times, each time relating another variable to $\eta$ and $p$, and the prognostic equations for $\rho^{(w)}$ and $T$ take the same form as in the optimal schemes discussed above.

The equations of state (75) and (76) must be solved simultaneously to obtain $p$ and $\eta$. For example

$$
\mathcal{L}_{2}(p) \equiv p-\left.\left.\frac{\partial p}{\partial \eta}\right|_{T} ^{(r)} \frac{\partial \eta}{\partial p}\right|_{\rho} ^{(r)} \overline{\bar{p}}=\left.\left.\frac{\partial p}{\partial \eta}\right|_{T} ^{(r)} \frac{\partial \eta}{\partial \rho}\right|_{p} ^{(r)} \bar{\rho}+\left.\frac{\partial p}{\partial T}\right|_{\eta} ^{(r)} T
$$

followed by back-substitution to obtain $\eta$. Again a vertical tridiagonal system must be solved. The operator $\mathcal{L}_{2}$ will be diagonally dominant and therefore invertible provided

$$
\left.\left.\frac{\partial p}{\partial \eta}\right|_{T} ^{(r)} \frac{\partial \eta}{\partial p}\right|_{\rho} ^{(r)}<1
$$

Again it may be verified that this condition does hold for a perfect gas.

Substituting (73) and (74) in (77) leads, after some manipulation, to

$$
-\mathrm{i} \omega \frac{\mathcal{L}_{2}(p)}{\rho^{(r)}}+c^{2} \mathcal{L}_{2}\left(\mathrm{i} k u+\mathrm{i} l v+\delta_{z} w-B \bar{w}\right)=0 .
$$

Provided $\mathcal{L}_{2}$ is invertible, this is equivalent to (34). The fact that the $p$ and $\rho^{(w)}$ tendency equations are of optimal form then means that the implied $\eta$ equation is of optimal form.

\section{Conclusions and Discussion}

Previous work has examined the ability of different vertical discretizations of the nonhydrostatic compressible Euler equations to capture accurately the propagation of acoustic, inertio-gravity, and Rossby waves. Here the approach has been extended to apply to a general equation of state, making it applicable to a wider range of geophysical fluid systems. This previous work had identified a number of choices of prognostic thermodynamic variables and vertical staggering (i.e. 'configurations') that give an optimal representation of wave propagation. The present work has identified several configurations that were previously thought to be suboptimal but which, when discretizated appropriately, can give optimal wave propagation after all.

The key idea behind the construction of the new optimal discretizations is to ensure that the corresponding linearized system is equivalent to the fundamental one that comes from the $(w \eta, u v p)$ configuration. Specifically, one must diagnose $\eta$ at $w$ levels from the predicted thermodynamic variables and diagnose $p$ at $u-v$ levels from the predcted thermodynamic variables. Then the buoyancy term in the $w$ equation must be expressed in terms of $\eta$ and $\bar{p}$. The evolution equation for $\eta$ must be expressed in advective form; the flux form would introduce additional averaging. If $\eta$ is not predicted then its implied evolution equation must be equivalent to the optimal advective form; this requirement constrains how the prognostic thermodynamic equations should be discretized. Similarly, if $p$ is not predicted then its implied evolution equation must be equivalent to the optimal form, in which the divergence involves no averaging.

Given a choice of two prognostic thermodynamic variables from $\rho, \eta, p$ and $T$, there are 12 possible configurations that have one thermodynamic variable at $w$ levels and one at $u-v$ levels. Of these 12, we have shown that 7 can be discretized in a way that gives optimal wave propagation. The remaining 5 involve the prediction of $\eta$ at $u-v$ levels or the prediction of $p$ at $w$ levels (or both). For these 5 there is no possibility to construct a discretization with an unaveraged $\eta$ at $w$ levels and an unaveraged $p$ at $u-v$ levels, as required for an optimal scheme. Thus, the 7 optimal discretizations identified here comprise essentially the complete set.

Some of the optimal discretizations identified here are less appealing than others from a practical point of view. For example, some do not predict the mass variable $\rho$, or predict it but not via a flux-form conservation equation (e.g. section 3.3), and so do not lend themselves to mass conservation. Others involve considerable extra complexity with no obvious compensating advantages (section 3.6, 3.7). Nevertheless, it is instructive to have this more complete picture of how vertical discretization affects wave propagation. Finally, it should be emphasized that linear wave propagation is but one consideration, albeit an important one, in the design of numerical models; others include the representation of hydrostatic and geostrophic balance (which is closely related to wave propagation), Lagrangian and integral conservation properties, and the representation of strongly nonlinear circulations.

\section{Acknowledgements}

I am grateful to Mike Bell and to Nigel Wood, both of the Met Office, for valuable discussions on vertical discretizations and their effect on wave propagation. The comments of three anonymous reviewers were helpful in improving the clarity of the presentation.

\section{References}

Akmaev RA. 2011. Whole atmosphere modeling: Connecting terrestrial and space weather. Rev. Geophys. 49(RG4004), doi:10.1029/2011/RG000364.

Charney JG, Phillips NA. 1953. Numerical integration of the quasi-geostrophic equations for barotropic and simple baroclinic flow. J. Meteorol. 10: 71-99.

Feistel R, Wright DG, Kretzschmar HJ, Hagen E, Herrmann S, Span R. 2010. Thermodynamic properties of sea air. Ocean Sci. 6: 91-141.

Fox-Rabinovitz MS. 1994. Computational dispersion properties of vertically staggered grids for atmospheric models. Mon. Wea. Rev. 122: 377-392.

Fox-Rabinovitz MS. 1996. Computational dispersion properties of 3D staggered grids for a nonhydrostatic anelastic system. Mon. Wea. Rev. 124: 498-510.

Girard C, Plante A, Desgagné M, McTaggart-Cowan R, Côté J, Charron M, Gravel S, Lee V, Patoine A, Qaddouri A, Roch M, Spacek L, Tanguay M, Vaillancourt PA, Zadra A. 2014. Staggered vertical discretization of the Canadian environmental multiscale (GEM) model using a coordinate of the log-hydrostatic-pressure type. Mon. Wea. Rev. 142: 1183-1196.

IOC, SCOR, IAPSO. 2010. The international thermodynamic equation of seawater-2010: Calculation and use of thermodynamic properties. Intergovernmental Oceanographic Commission, Manuals and Guides No. 56, URL http: / /www. TEOS-10.org.

Lesley LM, Purser RJ. 1992. A comparitive study of the performance of various vertical discretization schmes. Meteor. Atmos. Phys. 50: 61-73.

Lighthill J. 1978. Waves in Fluids. Cambridge University Press.

Liu Y. 2008. Impact of difference accuracy on computational properties of vertical grids for a nonhydrostatic model. Comput. Geosci. 12: 245-253.

Lorenz EN. 1960. Energy and numerical weather prediction. Tellus 12: 364 373.

Militzer B, Hubbard WB. 2013. Ab initio equation of state for hydrogenhelium mixtures with recalibration of the giant-planet mass-radius relation. Astrophys. J. 774(148), doi:10.1088/0004-637X/774/2/148.

Read PL, Thomas NPJ, Risch SH. 2000. An evaluation of Eulerian and semiLagrangian advection schemes in simulations of rotating, stratified flows in the laboratory. Part I: Axisymmetric flow. Mon. Wea. Rev. 128: 2835-2852.

Thuburn J. 2006. Vertical discretizations giving optimal representation of normal modes: Sensitivity to the form of the pressure-gradient term. Quart. J. Roy. Meteor. Soc. 132: 2809-2825.

Thuburn J, Wood N, Staniforth A. 2002. Normal modes of deep atmospheres. I: Spherical geometry. Quart. J. Roy. Meteor. Soc. 128: 1771-1792.

Thuburn J, Woollings TJ. 2005. Vertical discretizations for compressible Euler equation atmospheric models giving optimal representation of normal modes. J. Comput. Phys. 203: 386-404.

Tokioka T. 1978. Some considerations on vertical differencing. J. Meteor. Soc. Japan 56: 98-111. 
Toy MD, Randall DA. 2007. Comment on the article "vertical discretizations for compressible Euler equation atmospheric models giving optimal representation of normal modes" by Thuburn and Woollings. J. Comput. Phys. 223: 82-88.

Wood N, Staniforth A, White A, Allen T, Diamantakis M, Gross M, Melvin T, Smith C, Vosper S, Zerroukat M, Thuburn J. 2014. An inherently mass-conserving semi-implicit semi-lagrangian discretisation of the deepatmosphere global nonhydrostatic equations. Quart. J. Roy. Meteor. Soc. 140: $1505-1520$, doi:10.1002/qj.2235. 